LBNL- 51032

Paper presented at the

11th International Congress on Plasma Physics

Sydney, Australia, July 15-19, 2002

(and to be published in Plasma Physics \& Controlled Fusion)

\title{
GROWTH OF LARGE PATTERNED ARRAYS OF NEURONS USING PLASMA METHODS*
}

\author{
I.G. Brown, K.A. Bjornstad, E.A. Blakely, J.E. Galvin, O.R. Monteiro \\ and S. Sangyuenyongpipat ${ }^{\dagger}$ \\ Lawrence Berkeley National Laboratory \\ Berkeley, California 94720 USA
}

July 2002 
* This work was supported by the U.S. Department of Energy under Contract Number DE-AC03-76SF00098.

$\dagger$ Visiting scientist from the Department of Physics, Chiang Mai University, Chiang Mai 50200 ,

Thailand 


\title{
GROWTH OF LARGE PATTERNED ARRAYS OF NEURONS USING PLASMA METHODS
}

\author{
I.G. Brown, K.A. Bjornstad, E.A. Blakely, J.E. Galvin, O.R. Monteiro and \\ S. Sangyuenyongpipat \\ Lawrence Berkeley National Laboratory \\ Berkeley, California 94720, USA
}

\begin{abstract}
To understand how large systems of neurons communicate, we need to develop, among other things, methods for growing patterned networks of large numbers of neurons. Success with this challenge will be important to our understanding of how the brain works, as well as to the development of novel kinds of computer architecture that may parallel the organization of the brain. Large in vitro networks could show, for example, the emergence of stable patterns of activity and could lead to an understanding of how groups of neurons learn after repeated stimulation. We have investigated the use of metal ion implantation using a vacuum arc ion source, and plasma deposition with a filtered vacuum arc system, as a means of forming regions of selective neuronal attachment on surfaces. Lithographic masks created by treating surfaces with ion species that enhance or inhibit neuronal cell attachment allow subsequent proliferation and/or differentiation of the neurons to form desired patterns. Plasma deposition of optically transparent, electrically conducting, ultra-thin metal films can also be used to form electrodes for extra-cellular electrical stimulation of neurons. In the work described here we used glass microscope slides as substrates, and some of the experiments made use of simple masks to form patterns of ion beam or plasma deposition treated regions.
\end{abstract}


PC-12 rat neurons were then cultured on the treated substrates coated with Type I Collagen, and the growth and differentiation was monitored. Particularly good results were obtained, for example, for the case of plasma deposition of carbon to form a diamond-like carbon film of thickness about one hundred Angstroms. Neuron proliferation and the elaboration of dendrites and axons after the addition of nerve growth factor both showed excellent contrast, with prolific growth and differentiation on the treated surfaces and very low growth on the untreated surfaces. Here we describe our preliminary investigations, and summarize the results to date. 


\section{INTRODUCTION}

The study of the functional unit of the nervous system, the neuron, has been an active field of investigation for many years, both at the single-cell level, in vivo and in vitro, and at the level of large numbers of interconnected neurons, for example within the human brain [1]. The behavior of individual neurons has been studied using microelectrodes to monitor the electrical signals ("action potentials") generated within the neuron and along its dendrites (the branchlike arms that carry signals toward the neuron cell body where they are processed) and axons (the long "tail" that carries the neuron output signal to other cells). One can think of the single-cell electrical behavior as the performance at the "device level" [2], and at this level much is known. At the "system level", however, much less is known - we know very little about how large numbers of neurons communicate among themselves. There has been good progress made in the growth of neuron cultures in vitro. The neurons grow, extend dendrites and axons, form synapses, and create neural networks. In order to systematically explore the electrical characteristics of large numbers of associating neurons, however, we need first to develop techniques for forming 2-dimensional patterned arrays of large numbers of neurons. All of the parameters of the patterning should be under the control of and determined by the experimenter, including the geometry of the pattern, the line width, and the pattern size (number and density of neurons). The subsequent step is a daunting step indeed - we need then to discover and develop methods for monitoring the electrical activity throughout the array. Methods for monitoring the activity of small numbers of neurons have been developed, mostly making use of extracellular recording of the action potentials with extracellular microcircuit electrode arrays. Note that the use of chronic intercellular (impaled) microelectrodes (micron-size electrodes penetrating the cell wall) is not feasible because of cell death (the cell dies within a few hours of electrode insertion), and because the method cannot be extended to large arrays. It has not been possible up to now to adequately detect the 
spatial geography and temporal history of the action potentials in large neuronal arrays. Several approaches to patterning have been explored [3], including mechanical fabrication of troughs and ridges [4], laser micromachining [5], surface photochemical methods [6], photoresist methods, among others. These methods work and have been used to grow neural arrays. Here we describe some exploratory work that we have carried out investigating the suitability of vacuum-arc-plasma based methods of surface modification as a tool for forming large patterned neuronal arrays.

\section{EXPERIMENTAL}

Neuron cultures can be grown on glass or tissue culture plastic substrates coated with Type I Collagen. In the work described here we used ordinary glass microscope slides of dimension 1 " $\mathrm{x} 3$ ". In the first part of the work, our goal was to explore the effects on neuron growth of either (i) metal ion implantation into the glass, or (ii) plasma deposition onto the glass surface.

Ion implantation was done using a vacuum arc ion source ("Mevva V")-based system that has been fully described elsewhere [7-9]. In this facility, a high current, energetic metal ion beam is formed by a vacuum arc ion source and used to do high energy ion implantation. The ion beam is about $10 \mathrm{~cm}$ diameter at the extractor, of ion energy up to one or two hundred $\mathrm{keV}$, and of (pulsed) ion current typically a few hundred milliamperes. The source is operated in a repetitively pulsed mode, with pulse length $250 \mu$ s and pulse repetition rate a few pulses per second. The metal ion species can be any of the solid metals of the periodic table as well as metallic compounds and alloys. Since the ions generated by the vacuum arc are in general multiply stripped, for a given extraction voltage the ion beam can have a mean energy of up to about $200 \mathrm{keV}$ or more with discrete ion energy components up to about $500 \mathrm{keV}$. Vacuum 
arc ion sources have been used for ion implantation application by a growing number of workers at many different laboratories around the world [10]. Implantation is done in a broadbeam mode, without magnetic analysis of charge-to-mass components, and the ion trajectories are line-of-sight from ion source to target. We note that implantation into insulating target materials, such as glass, is readily accomplished in this kind of system without any charge build-up problems; we have shown in prior work [11] that the Mevva-produced high current metal ion beam provides self-neutralization of insulating targets via the background cold electron sea formed by the ion beam itself, alleviating the need for any additional electron source for target charge neutralization. For the present work, the implants were done at a relatively low energy of about 10 to $30 \mathrm{keV}$, with doses from $5 \times 10^{14}$ to $1 \times 10^{16} \mathrm{~cm}^{-2}$, and with several different ion species including $\mathrm{C}, \mathrm{Mg}, \mathrm{Al}$, Ti and Ta.

Plasma deposition was done using a filtered vacuum arc system that has been described in detail elsewhere $[9,12,13]$. The vacuum arc (or cathodic arc) is a high current discharge between two electrodes in vacuum [13]. Metal (or carbon) plasma is produced in abundance from the cathode material, and it is this plasma that carries the arc current. For the work described here a repetitively pulsed vacuum arc plasma source was used; the pulse length was $5 \mathrm{msec}$ and the repetition rate was 1 pps. Along with the metal plasma that is generated by the vacuum arc a flux of macroscopic droplets of size in the broad range $0.1-10$ microns is also produced, and we routinely use a $90^{\circ}$ magnetic filter for their removal - a curved 'magnetic duct' which stops line-of-sight transmission of macroparticles while allowing the transmission of plasma by virtue of an axial magnetic field which ducts the plasma through the filter [14]. The glass substrate was mounted on a grounded holder positioned about $10 \mathrm{~cm}$ from the duct exit. A simplified schematic of the filtered vacuum arc plasma deposition system is shown in Figure 1. Films were in this way formed on the glass microscope slides, of thickness in the approximate range $30-300 \AA$. Ion species used, and thus the kinds of film materials formed, 
included $\mathrm{C}, \mathrm{Mg}$, Ti, Pd, Ta, Ir, Pt and Au. By doing the depositions at a somewhat elevated background pressure it is straightforward to form metal oxides, and thus we also made films of aluminum oxide, titanium oxide and tantalum oxide. A characteristic feature of vacuum-arcproduced plasmas is the relatively high directed energy with which the ions are formed, in the approximate range 20 to $150 \mathrm{eV}$ depending on the ion species [15]. The film deposition is thus an energetic deposition, and for the case of carbon this results in the film material formed being a high quality, hydrogen-free, diamond-like carbon (DLC) [16,17], not amorphous carbon or graphite. As described below, we found that the carbon films were particularly advantageous for enhanced neuron growth.

We obtained PC-12 neurons from the American Type Culture Collection (Manassas, VA). The PC-12 cell-line was derived from a transplantable rat pheochromocytoma from the adrenal gland. The cells are grown in RPMI 1640 media with 2 gm/L glucose (Invitrogen), 10\% heatinactivated horse serum (Invitrogen), 5\% fetal bovine serum (HyClone), $2 \mathrm{mM}$ L-glutamine, $1.5 \mathrm{~g} / \mathrm{L}$ sodium bicarbonate, pen strep at $37^{0} \mathrm{C}, 7.5 \% \mathrm{CO}_{2}$ on Type I Collagen coated Biocoat ${ }^{\mathrm{TM}}$ (Becton Dickinson) plastic $100 \mathrm{~mm}$ petri plates. Stock cultures were fed every three days with 2/3rds fresh media, and subcultured every 9 days with a 1:4 cell split ratio. Nerve Growth Factor (NGF) $2.5 \mathrm{~S}$ (Invitrogen) was added to cell densities at concentrations of $50 \mathrm{ng} / \mathrm{ml}$. On a collagen-coated substrate, neurite elongation proceeds at an average rate of $\sim 50 \mu \mathrm{m} /$ day for at least 10 days. After 2 weeks of NGF exposure, the cultures generate a dense mat of neuritic processes. Generally, at least $90-95 \%$ of the cells in the cultures produce neurites.

PC-12 cells were inoculated onto pre-cleaned, plasma-deposited DLC-coated, Type I Collagen-coated sterile glass slides at $1 \times 10^{5}$ cells $/ \mathrm{ml}$. Cells were allowed to adhere to the slide in a $7.5 \% \mathrm{CO}_{2}$ incubator at $37^{0} \mathrm{C}$, for 3 hours, and then gently flooded with growth media. Cell growth was monitored by phase light microscopy. Cells were photographed with 
a digital Spot Camera on a Nikon TMS scope using the Spot Advanced software, and printed using Adobe Photo Shop. After 3-6 days of cell growth, NGF was added to the media at 50 $\mathrm{ng} / \mathrm{ml}$. After the addition of NGF, cell division stops and differentiation begins. PC-12 cells double every 96 hours. Cultures were visually monitored daily and images captured every other day, up to 1.5 months after initiation of the cultures.

\section{RESULTS}

Neurons grew on all the processed substrates, but there was a wide variation observed in the total number of attached cells and their morphology. Under identical neuron growth conditions for each substrate surface tested, the neuronal cell density attained in the cultures was found to vary over many orders of magnitude for the various processing methods investigated. That is, we can state unequivocally that processing of the glass substrate in the ways described above does indeed provide a means of controlling the neuron growth. Ion implantation, for the entire range of parameters explored, was found to be universally poor in its effect - the growth rate and the culture cell density at all times during the growth were both low compared to the case of plasma deposition. For example, glass slides that had been ion implanted with carbon under a range of conditions, including at particularly low energy (10 $\mathrm{keV})$ so as to form a carbon profile close to the glass surface, and at relatively high dose ( $1 \mathrm{x}$ $10^{16} \mathrm{~cm}^{-2}$ ) so as to increase the surface carbon concentration, also yielded unimpressive results. We quickly abandoned further exploration of ion implantation as a tool for enhanced neuron growth.

Plasma deposition, on the other hand, was seen to provide significantly enhanced neuron growth for some kinds of film materials (plasma deposition species). We found that the metals 
provided a generally positive growth enhancement and that all of the metal oxides were generally negative in their effect. The single film material that stood out as providing vastly enhanced growth was carbon, which as described above is deposited in the form of hydrogenfree diamond-like carbon, or DLC. We therefore chose to investigate neuron growth on carbon surfaces in more detail. Variation of DLC film thickness indicated that a film thickness of about 100 - $150 \AA$ was near optimum. For thinner films, the neuron "contrast ratio" - ratio of neuron growth density on the DLC-coated region to density on the non-DLC-coated region - was less, and thicker films tended to delaminate from the substrate.

The photograph in Figure 2 shows clearly how neurons grew preferentially on a DLC coated substrate. One can see that (i) neuron growth is healthy on the upper DLC-coated region, with virtually no growth on the lower uncoated region, (ii) in the region of good growth, the DLC region, neurons grow extended processes (axons and neurons), (iii) the neuron extensions show a pronounced tendency to confine their growth to the DLC region.

The results of another growth experiment are shown in Figure 3. Here the neuron density is prolific, much greater than would be chosen for a controlled experiment. But the point is made beautifully clear that the growth is limited to only the DLC-coated region. Neuron growth is on a glass substrate processed by plasma deposition of $\sim 100 \AA$ coating of diamondlike carbon (DLC) film. The plasma deposition was such that the lower part of each photo is the DLC-treated region, and the upper part is not DLC-treated. The entire substrate was collagen coated, and the neurons were seeded over the entire surface. The left-hand photo shows the delicate neurite growth that develops on the DLC-treated region; the right-hand photo shows that the neuron growth in the DLC-treated region continues to a dense and prolific neuron density. These results indicate that neurons grow selectively on the lower DLC-treated regions and not on the upper untreated regions. The contrast (ratio of neuron 
density in the treated region to neuron density in the untreated region) is very high, and neuron growth in the treated region is healthy.

The results of our first attempt at neuron patterning are shown in Figure 4. Neuron growth is on a glass substrate processed by plasma deposition of $\sim 150 \AA$ diamond-like carbon (DLC) film. Prior to deposition, "LBNL" was written on the glass slide using a fine marker pen, and then the DLC deposition was carried out. After DLC deposition, the ink was removed with alcohol, thus leaving "LBNL" patterned in negative in the DLC film. The slide was then coated with Type I Collagen and seeded with PC-12 rat neurons. The neurons were allowed to grow for 3 days, at which point NGF (Nerve Growth Factor) was added. The micrographs shown in Fig. 3 were taken after a growth period of 6 days after initiation of the cultures.

\section{CONCLUSIONS}

The work described here has demonstrated the suitability of filtered-vacuum-arc deposition methods for forming patterned arrays of large numbers of live neurons. We have shown that energetic plasma deposition of carbon to form an ultra-thin layer of amorphous diamond on the substrate surface provides a means for selective neuron attachment, growth, and differentiation

on that surface. The optimal DLC thickness for neuronal patterning is $\sim 100 \AA$ to $150 \AA$. The neuron growth contrast (ratio of neuron density on plasma-treated regions to neuron density on untreated regions) can be very high. The deposited carbon films are strong and stable, and remain intact on the glass substrate for periods of at least 2 months.

Acknowledgements - One of us (S.S.) is indebted to the Royal Golden Jubilee program of the 
Thailand Research Fund for support at the Lawrence Berkeley National Laboratory. This work was supported by the U.S. Department of Energy under Contract Number DE-AC03$76 \mathrm{SF} 00098$. 


\section{REFERENCES}

1. Vaudry D., Stork P. J. S., Lazarovici P. and Eiden L. E. (2002) Science 296, 1648.

2. Wilkinson C. and Curtis A. (1999) Physics World 12, 45.

3. Stenger D. A. and McKenna T. M. (1994). Enabling Technologies for Cultured Neural Networks. Academic Press, San Diego.

4. Miller C., Shanks H., Witt A., Rutkowski G. and Mallapragada S. (2001) Biomaterials $22,1263$.

5. Corey J. M., Wheeler B. C. and Brewer G. J. (1991) J. Neurosci. Res. 30, 300.

6. Hickman J. J., Bhatia S. K., Quong J. N., Shoen P., Stenger D. A., Pike C. J. and Cotman C.W. (1994) J. Vac. Sci. Tech. A12, 607.

7. Brown I. G. (1994) Rev. Sci. Instrum. 65, 3061.

8. Brown I. G., Dickinson M. R., Galvin J. E., Godechot X. and MacGill R. A. (1992) Surf. Coat. Technol. 51, 529.

9. Brown I. G., Anders A., Dickinson M. R., MacGill R. A. and Monteiro O. R. (1999) Surf. Coat. Technol. 112, 271.

10. See, for instance: Proceedings of the biennial conferences on Ion Beam Modification of Materials (IBMM), published in Nucl. Instr. and Meth.; Proceedings of the biennial conferences on Surface Modification of Metals by Ion Beams (SMMIB), published in Surf. Coat. Technol.; Rev. Sci. Instrum. (1994), 65, pp. 3061-3139, (this journal issue contains a review of vacuum arc ion sources and also a collection of papers on this topic $\begin{array}{ll}\text { by a number } & \text { of authors from }\end{array}$ laboratories around the world).

11. Liu F., Monteiro O. R., Yu K. M. and Brown I. G. (1997) Nucl. Instrum. Meth. B132, 188.

12. Anders S., Anders A. and Brown I. G. (1993) J. Appl. Phys. 74, 4239. 
13. Boxman R. L., Martin P. J. and Sanders D. M. (1995), editors, Vacuum Arc Science and Technology. Noyes, New York.

14. Anders A., Anders S. and Brown I. G. (1994) J. Appl. Phys. 75, 4900.

15. Anders A. and Yushkov G. Yu (2002) J. Appl. Phys. 91, 4824.

16. Pharr G. M., Callahan D. L., McAdams S. D., Tsui T. Y., Anders S., Anders A., Ager J. W., Brown I. G., Bhatia C. S., Silva S. R. P. and Robertson J. (1996) Appl. Phys. Lett. 68, 779.

17. Monteiro O. R. (1999) Nucl. Instrum. Meth. Phys. Res. B148, 12. 


\section{FIGURE CAPTIONS}

Fig. 1 Simplified schematic of the filtered vacuum arc plasma deposition set-up.

Fig. 2 Selective neuron growth on DLC-coated substrate.

Neuron growth after 15 days on a glass slide onto which a $100 \AA$ A thick film of DLC was deposited. The DLC region can be seen as a slightly darker region occupying the upper $75 \%$ of the whole region viewed; there was no DLC coating on the lower part of the image. The whole slide was coated with Type I Collagen.

Fig. 3 Selective PC-12 neuron growth on collagen-coated, DLC-plasma-processed surface.

The lower part of each photograph shown was DLC coated, with the upper part not coated; the substrate was collagen coated, and neurons were then seeded over the entire surface. A delicate neurite growth develops on the DLC-treated region (lefthand photo), which develops into a dense and prolific neuron field (right-hand photo). (Scale: the width of each photograph is several hundred microns).

Fig. 4 Patterned growth of neurons to form "LBNL" (in negative). 
Figure 1 
QuickTime
Photo - JPEG decompressor
are needed to see this picture.

Figure 2 


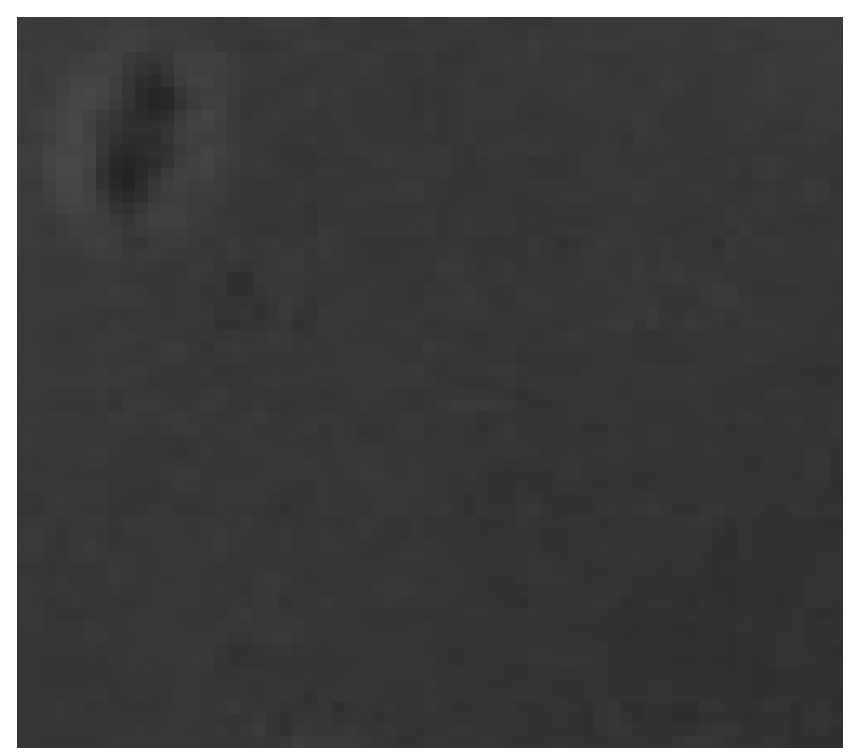

Figure 3 
Figure 4 